\title{
Gene Silencing Genetic Algorithm for 0/1 Knapsack with Object Preferences
}

\author{
S. Siva Sathya \\ Department of Computer Science, Pondicherry University, \\ Puducherry, India \\ E-mail: ssivasathya@gmail.com \\ S. Kuppuswami \\ Department of Computer Science, Pondicherry University, \\ Puducherry, India \\ S. Sendhil Kumar \\ IBM India \\ Bangalore, India \\ Received 16 July 2009 \\ Accepted 13 August 2010
}

\begin{abstract}
Genetic Algorithms are efficient search and optimization techniques inspired by natural evolution. To show the difficulties in solving constrained optimization problems through GA, the $0 / 1$ knapsack problem with user specific object preferences has been taken up. A new genetic operator, namely, 'gene silencing' inspired from biology is used along with standard GA. The experimental results for varying number of objects and user preferences show that genetic algorithm with gene silencing produces better results when compared to standard GA.
\end{abstract}

Keywords: Genetic algorithm, 0/1 knapsack, constraint satisfaction, gene silencing operator 


\section{Introduction}

Genetic Algorithms ${ }^{1,2}$ are generally considered to be good at solving optimization problems. But, constrained optimization through GA is considered to be a challenging task because, GAs are not considered to be directly suitable to solve such problems. To illustrate the fact, the problem of $0 / 1$ knapsack with multiple user specific object preferences has been taken up in this paper. This is a slight variant of the standard $0 / 1$ knapsack problem wherein, the user is free to select some objects of his choice that should be compulsorily included in the knapsack also satisfying the problem constraints. This can be stated as an instance specific constraint as it can be varied with each execution of the problem. As standard GA was not able to produce the required solutions in the expected time period, we attempted for GA with population sizing and fitness function variation, ${ }^{3,4}$ which incorporates the reward score for expected object preferences. ${ }^{5}$ But still, the results were not very satisfactory. Hence, we have incorporated a new operator called gene silencing inspired from biology to meet out the required object preferences in this paper.

The organization of the paper is as follows: Section-2 gives the related work; section-3 explains the proposed system which includes a description of the new operator namely gene silencing and how it is used along with GA. Section-4 gives the experimental results and section- 5 concludes the paper.

\section{Related Work}

A number of Genetic Algorithms have been used to solve constraint optimization problem and ${ }^{6,7}$ are some of them that are used for solving knapsack with multiple constraints.

Methodologies in literature include different forms of penalty functions ${ }^{8,9}$ that vary with the problem. Since the penalty factors have to be assigned based on the degree of violation of the constraints, it seemed to be a difficult task. Also, the issue of diversity maintenance along with constrained optimization has to be taken care as explained in $^{10}$ to get a globally optimum solution. This paved the way for identifying a new biologically inspired operator for solving the constrained optimization problems. Other biologically inspired operators like transposons ${ }^{11}$, transformation ${ }^{12}$, etc, have also been applied to genetic algorithms in the past for various reasons and they were found to yield promising results. With respect to the gene silencing operator, some related operators have been found in literature. For instance, the mask operator ${ }^{13}$ is a type of operator that works at the gene level. This is used along with crossover operator to set some preferences in the selection of genes during crossover, but the problem with mask is the selection of a suitable mask that varies with problem. . Moreover, it is not heritable over the successive generations. This will result in the disruption of the good building block by the crossover and mutation operators.

Similarly, some repair functions ${ }^{14}$ have also been used at the gene level, even for solving the 0/1 knapsack problem. Some knowledge based operators ${ }^{15}$ are also used in other type of problems like course timetabling problem to set some user specific preferences. Also, standard GA would set different penalty values ${ }^{16}$ based on the requirement. A variety of repair functions and crossover operators have also been applied to the above said problem. Alternatively, it has been shown that based on biased genetic operators $^{17}$, one could achieve the required preference settings. But all the above stated techniques have got some limitations like the following.

- One may not get the exact solution that is required.

- If there are one or more preferences you may not get the correct choice of preference on convergence.

- Choosing the right penalty values for instance specific constraint is difficult and the success of the GA relies on the efficient determination of these penalty values.

- When instance specific constraints are satisfied, there may be a few violations of high penalty constraints or a sacrifice in the maximum fitness value that could be achieved.

- The number of generations taken for convergence increases drastically with the number of preferences, and sometimes, the GA may not converge at all if $100 \%$ preference settings are expected. 
Thus, this paper presents a different type of knapsack problem with user specific object preferences, for which no specific work is reported in literature.

\section{Gene Silencing Genetic Algorithm for the 0/1 Knapsack Problem (with object preferences)}

This section explains about the new operator namely gene silencing, its usage in genetic algorithm and its applicability to the $0 / 1$ knapsack problem.

\subsection{Gene Silencing}

Genes are considered to be small segments of DNA housed in chromosomes and each gene is responsible for a specific function. ${ }^{18,19,20}$ Among the several thousand functional genes present in the human body, not all the genes are active at a given instance. Most of the genes are turned off or silenced appropriately preventing from doing their work of protein synthesis. For example, thousand of genes are active only during embryo development, but remain silent in healthy adults. Similarly, certain genes have to be activated in certain parts of the body and silenced in others where its functionality is not required. Example, some genes have to be activated in skin and silenced in the heart, liver and other organs.

The most important observation made is that, the phenomenon of gene silencing is heritable, i.e., when a cell divides, its daughter cells maintain not only copies of its DNA, but also the silencing of these genes. Lot of research is now being carried out in this field of genetics and sufficient knowledge of this mechanism could be exploited to evolve new cancer therapies aimed at resilencing inappropriately activated genes. Gene silencing could also be used for determining gene function.

\subsection{GA with Gene Silencing}

The principles of genetic algorithm mimic the process of natural evolution. The concept of gene silencing discussed in the previous section could be adopted in genetic algorithm as an operator where other natural operators like crossover and mutation are already being applied for any standard GA. The population in GA comprises of the chromosomes or individuals. Each chromosome is again composed of genes, which are the target of the gene silencing operator. This property is particularly useful to set certain user specific constraints or preferences that are present in some problem apart from their optimization criterion. Hence this operator works at the gene level. This operators functions as follows: Whenever a chromosome is encountered with the required gene position set according to the user preference, they are marked as silenced. This helps is the preservation of the required building blocks to obtain an optimal solution. Once silenced, the crossover and mutation operators do not affect the particular genes. As this property of gene is heritable in nature, they are preserved over successive generations. Normally, crossover operator disrupts the good building blocks or genes, even though they are considered to be useful to evolve good solutions. But through gene silencing, the required genes are preserved from the disruptions of crossover or mutation, allowing crossover and mutation to evolve an optimal solution with the other parts of the chromosome. Since the other parts of the chromosome undergo normal crossover and mutation the application of gene silencing to the required genes in the chromosome will not necessarily lead the Genetic algorithm towards a biased solution tending to produce local optima. To evolve the optimal solution the following steps are to be followed.

(i) Generate the initial population.

(ii) Evaluate the fitness as per general $0 / 1$ knapsack problem.

(iii) Apply gene silencing operator to the individuals who satisfy the object preferences.

(iv) (The operator will silence the required gene positions corresponding to the user specified object positions)

(v) Apply the other genetic operators (selection, crossover and mutation) to the individuals in the current generation.

(vi) Pass the generated offspring to the next generation.

(vii)Go to step-(ii)

Gene silencing could be mathematically represented as follows: Let $\mathrm{Ci}=\left\{\mathrm{g}_{\mathrm{i} 1}, \mathrm{~g}_{\mathrm{i} 2}, \ldots . \mathrm{g}_{\text {in }}\right.$ \}be the set of all genes in chromosome-i and $\mathrm{Cj}=\left\{\mathrm{g}_{\mathrm{j} 1}, \mathrm{~g}_{\mathrm{j} 2}, \ldots \mathrm{g}_{\mathrm{jn}}\right\}$ be the set of all genes in chromosome-j.

The consequence of silencing is very much evident during the process of crossover and mutation over successive generations. Because 
the property of gene silencing is heritable in nature, the silenced gene does not take part in the process of crossover or mutation. This is shown in the figure below. Assume a single point crossover and the cut point is chosen to be some $\mathrm{k}$ in a single point crossover. This implies that the genetic materials of chromosomes $\mathrm{Ci}$ and $\mathrm{Cj}$ get exchanged after this cut point $\mathrm{k}$. Assume that the $(\mathrm{k}+1)^{\text {th }}$ gene in both the chromosome-i and chromosome- $\mathrm{j}$ are the instance preferences and they are to be silenced. The crossover operation between chromosome- $i$ and chromosome- $j$ is represented as follows. The below figure shows that though the crossover point is at position $\mathrm{k}$, and the genetic materials are exchanged from the kth position, the genes $g_{i k+1}$, and $g_{j k+1}$, remains unaffected due to crossover. As gene silencing is heritable, this setup remains unaffected over successive generations, thus preserving the individual preferences. Similar arguments can be had with mutation operator also.

\section{Experimental Results}

To examine the performance of our approach for problems with instance specific constraints, the 0/1 knapsack problem ${ }^{21}$, a special kind of knapsack problem belonging to the class of NP hard problems has been chosen. Here, the 0/1 knapsack problem with varying percentage of user specific object preferences has been taken up. The modified 0/1 knapsack problem can be stated as follows:

Let $\mathrm{n}$ be the number of given objects and $\mathrm{w}_{\mathrm{i}}$ be the weight of the $i^{\text {th }}$ item, $p_{i}$ be the profit accrued when the $i^{\text {th }}$ item is carried in the knapsack, and $\mathrm{C}$ be the capacity of the knapsack. Let $\mathrm{x}_{\mathrm{i}}$ be a variable, the value of which is either zero or one. The variable $x_{i}$ has the value one when the $i^{\text {th }}$ item is carried in the knapsack and zero otherwise.

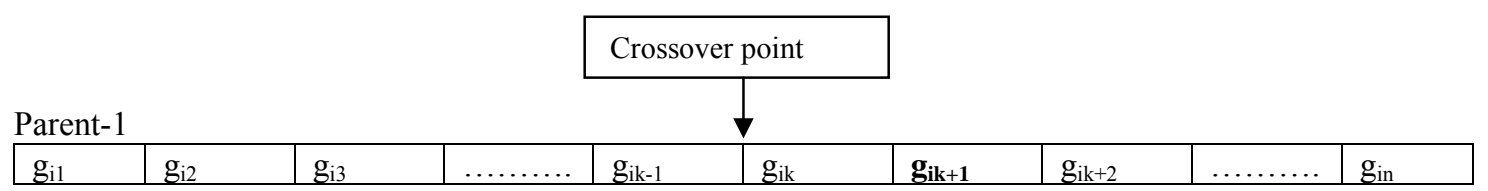

Parent-2

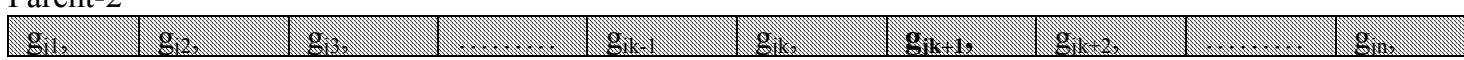

After crossover:

Child-1

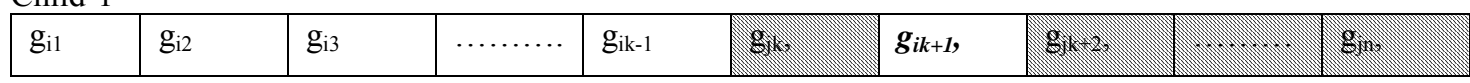

Child-2

\begin{tabular}{|c|c|c|c|c|c|c|c|c|c|}
\hline & $g_{12}$ & $g_{3}$, & 20.20 .20 & $g_{i k-1}$ & $\mathrm{~g}_{\mathrm{ik}}$ & $g_{i k+1}$ & $\mathrm{~g}_{\mathrm{ik}+2}$ & ......... & $g_{j}$ \\
\hline
\end{tabular}

Figure 1- Effects of gene silencing after crossover 
Given $\left\{\mathrm{w}_{1}, \mathrm{w}_{2}, \ldots . \mathrm{w}_{\mathrm{n}}\right\}$ and $\left\{\mathrm{p}_{1}, \mathrm{p}_{2}, \ldots . \mathrm{p}_{\mathrm{n}}\right\}$, our objective is to

maximize $f\left(x_{1}, \ldots \ldots ., x_{n}\right)=\sum_{j=1}^{n} p_{j} x_{j}$

subject to the constraint,

$\sum_{j=1}^{n} w_{j} x_{j} \leq c, \quad x_{j}=0$ or $1, \quad j=1, \ldots \ldots, n$

The above constraints are specific to the $0 / 1$ knapsack problem. In addition to these constraints, consider an instance specific constraint. Denote by $\mathrm{S}$, the set of above said variables $\mathrm{x}_{\mathrm{i}}$. Let $\mathrm{A}$ denote the set of user preference objects, $A=\{$ User preference objects $\}$. Then ACS and let some $x_{j} \in A$, we have to consider the following constraint.

$\mathrm{f}\left(\mathrm{x}_{1}, \mathrm{x}_{2}, \ldots \ldots \mathrm{x}_{\mathrm{n}}\right)$ attains the maximum value subject to

$\sum_{j=1}^{n} w_{j} x_{j} \leq c, \quad x_{j}=0$ or $1, \quad j=1, \ldots \ldots, n$

and $x_{j}=1$ for such of those $x_{j} \in A$

this modified form of $0 / 1$ knapsack consists of user specific object preferences that vary with every instance of GA execution, it has been used to test the effectiveness of the gene silencing GA.

The chromosome representation that is used for the $0 / 1$ knapsack problem is a bit vector consisting of zeroes and ones. Each gene here refers to an object $i$ that is either 1 or 0 depending on the inclusion or noninclusion of an object $\mathrm{i}$ into the knapsack respectively.

This being the general 0/1 knapsack problem, which can be solved by any standard GA, this paper assumes some object preferences. Hence some percentage of xi, are strictly required to be 1 , which means the strict inclusion of certain objects in the knapsack as specified by the user. This varies for each problem instance and hence cannot be easily achieved by standard GA through random iteration and standard genetic operators.

The input data was generated randomly with the following parameter settings. The problem size was varied with the number of objects being 100, 200, 300,
400 ,etc. The values of $\mathrm{Wi}$ and $\mathrm{Pi}$ was randomly chosen within the range (1..100). The knapsack capacity $\mathrm{C}$ was each time chosen to be less than or half of the total weight of all the $n$ objects. This was to ensure that there are few objects in the knapsack with more number of possible combinations. Thus the capability of GA with gene silencing could be thoroughly explored rather than an incidental occurrence of $1 \mathrm{~s}$ in the preferred genes. The percentage of object preferences is obtained from the user for each GA execution. Results were obtained for varying percentages of object preferences. A set of experiments were carried out to evaluate the performance of our approach with the standard GA with and without the gene silencing operator. Each experiment comprises of about 50 runs.

The results of only four experiments, each with five runs is given in the subsequent pages due to space limitations. The experiments were carried out for a fixed number of generations and the settings of the GA parameters are as follows:

Population size : 100;

Selection : Roulette Wheel

Elitism : 0.10

Crossover percentage $\mathrm{P}_{\mathrm{c}} \quad: 0.85$,

Crossover type : single point

Mutation Probability $\mathrm{P}_{\mathrm{m}}$ : 0.05 ,

Mutationtype : Swap Mutation.

No. of generations : 100

Table 1. Experiment-1 with 100 Objects and Preference of 5 $\%$ and $10 \%$

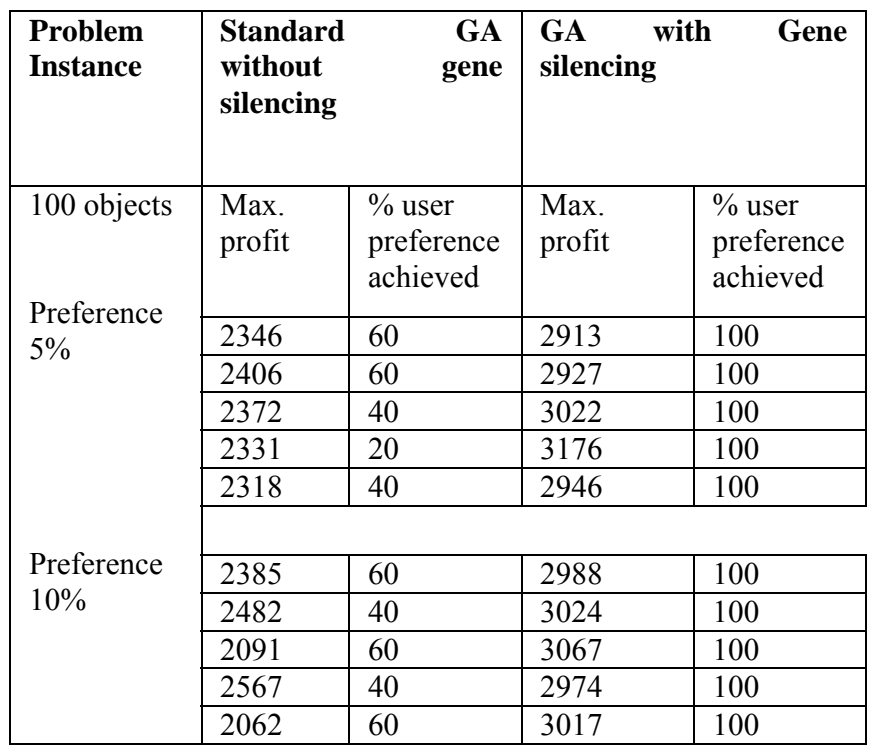


Table 2. Experiment-2 with 200 Objects and Preference of $5 \%$ and $10 \%$

\begin{tabular}{|c|c|c|c|c|}
\hline \multirow{2}{*}{$\begin{array}{c}\text { Problem Instance } \\
200 \text { objects }\end{array}$} & \multicolumn{2}{|c|}{$\begin{array}{l}\text { Standard GA without gene } \\
\text { silencing }\end{array}$} & \multicolumn{2}{|c|}{ GA with Gene silencing } \\
\hline & $\begin{array}{l}\text { Max. } \\
\text { profit }\end{array}$ & $\begin{array}{c}\% \text { user } \\
\text { preference } \\
\text { achieved }\end{array}$ & Max. profit & $\begin{array}{c}\% \text { user preference } \\
\text { achieved }\end{array}$ \\
\hline \multirow{4}{*}{ Preference $5 \%$} & 4365 & 40 & 5749 & 100 \\
\hline & 5025 & 30 & 5679 & 100 \\
\hline & 3702 & 50 & 5437 & 100 \\
\hline & 4186 & 30 & 5582 & 100 \\
\hline \multirow{7}{*}{ Preference $10 \%$} & 4762 & 40 & 5623 & 100 \\
\hline & & & & \\
\hline & 4353 & 40 & 5815 & 100 \\
\hline & 5264 & 40 & 5951 & 100 \\
\hline & 4980 & 20 & 6023 & 100 \\
\hline & 4667 & 70 & 5812 & 100 \\
\hline & 4876 & 70 & 5786 & 100 \\
\hline
\end{tabular}

Table 3. Experiment-3 with 300 Objects and Preference of $5 \%$ and $10 \%$

\begin{tabular}{|c|c|c|c|c|}
\hline \multirow{2}{*}{$\begin{array}{c}\text { Problem Instance } \\
300 \text { objects }\end{array}$} & \multicolumn{2}{|c|}{$\begin{array}{l}\text { Standard GA without } \\
\text { gene silencing }\end{array}$} & \multicolumn{2}{|c|}{ GA with Gene silencing } \\
\hline & $\begin{array}{l}\text { Max. } \\
\text { profit }\end{array}$ & $\begin{array}{l}\% \text { user } \\
\text { preference } \\
\text { achieved }\end{array}$ & Max. profit & $\begin{array}{c}\% \text { user preference } \\
\text { achieved }\end{array}$ \\
\hline \multirow[t]{5}{*}{ Preference $5 \%$} & 8441 & 60 & 9001 & 100 \\
\hline & 8203 & 50 & 8840 & 100 \\
\hline & 8161 & 60 & 9168 & 100 \\
\hline & 8207 & 70 & 9015 & 100 \\
\hline & 8285 & 60 & 8971 & 100 \\
\hline \multicolumn{5}{|l|}{ Preference $10 \%$} \\
\hline & 9133 & 70 & 9797 & 100 \\
\hline & 9288 & 80 & 9816 & 100 \\
\hline & 8871 & 60 & 9648 & 100 \\
\hline & 8774 & 70 & 10092 & 100 \\
\hline & 9476 & 70 & 9903 & 100 \\
\hline
\end{tabular}


Table 4. Experiment-4 with 400 Objects and Preference of $5 \%$ and $10 \%$

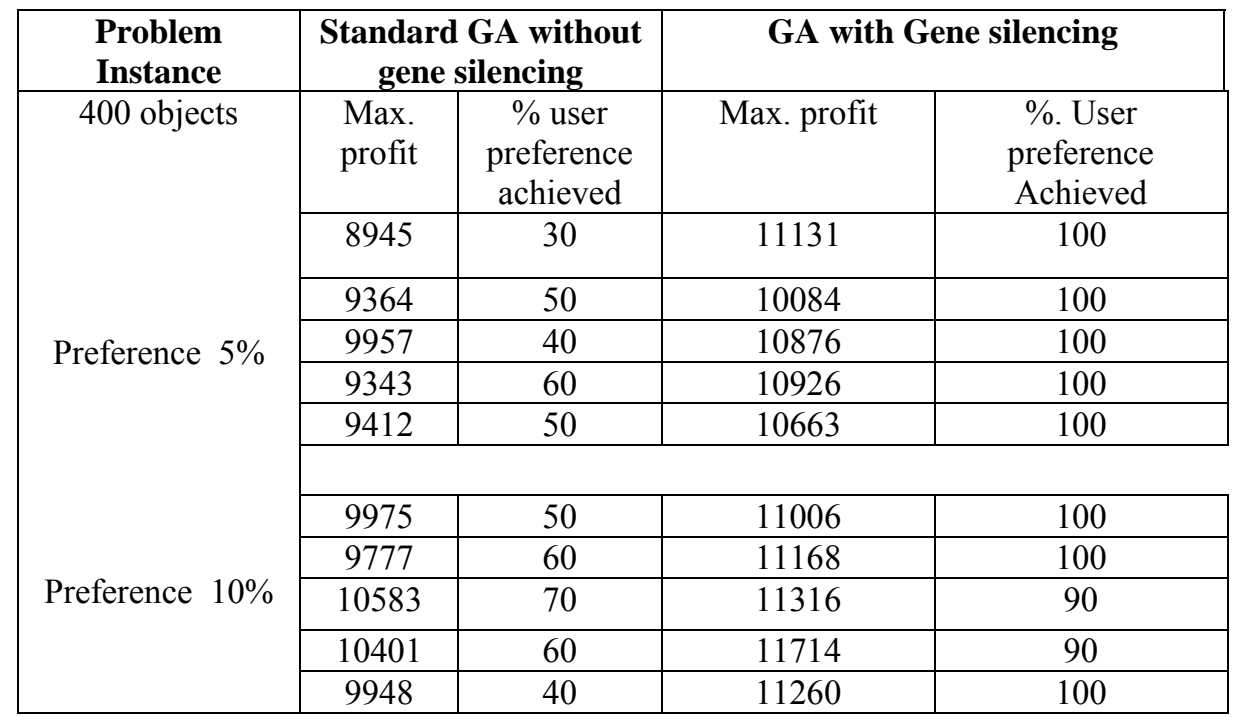

From the experimental results it is found that SGA fails to satisfy all user specified object preferences whereas GA with gene silencing achieves $100 \%$ object preferences most of the time the GA was executed. For example, $10 \%$ object preference for 400 objects implies that there are 40 user specific object preferences that have to be included in the knapsack compulsorily in addition to satisfying the profit weight constraint of the $0 / 1$ knapsack problem. This is very time consuming and cumbersome with the standard GA if it was allowed to run till convergence. Most of the time, it did not converge with the required preference setting. Hence, the number of generations was fixed up uniformly and the results compared for both the methods.

\section{Conclusion and Future Enhancements}

It has been concluded that for problems like $0 / 1$ knapsack (with object preferences), standard GA fails to converge with the required preference settings. If standard GA is allowed to run for a fixed number of generations, the user specified object preferences are not satisfied completely $(100 \%)$. Hence a new gene silencing operator that is heritable over generations has been applied. The results show that it is very helpful to achieve a high percentage of user specific object preferences without sacrificing the profits gained in the knapsack. Research is under progress for the tuning of control parameters (selection, crossover, mutation methods and their percentages) to evolve best results.

\section{References}

1. David E. Goldberg, Genetic Algorithms in Search, Optimization, and Machine Learning, Pearson Education, Ninth edition, 2005.

2. Mitchell Melanie, An introduction to Genetic Algorithms, A Bradford Book, The MIT press, Fifth printing, 1999.

3. Arabas J, Michalewicz Z, Mulawka J, GAVaPS - a genetic algorithm with varying population size', In Proc. of the First IEEE Conf. on Evolutionary Computation, pp. 73-78. IEEE Press, 1994.

4. Smith R. E, Smuda E, Adaptively resizing populations: Algorithm, analysis, and first results', Complex Systems, 9:47-72, 1995.

5. S.Siva Sathya, S.Kuppuswami, Vivek Ranjan, Rajeev Kumar Singh, (2008), Genetic Algorithm with Population sizing \& Reward function for $0 / 1$ knapsack with object preferences, In Proceedings of the International conference on Information Processing, 2008, Bangalore, India, pp. 594-603.

6. Anabela Simoes, Ernesto Costa, An Evolutionary Approach to the zero/one knapsack problem:Testing ideas from biology, In Proceedings of the Fifth International Conference on Artificial Neural Networks and Genetic Algorithms (ICANNGA'2001), Prague, Czech Republic, 22-25 April, 2001. 
7. J.Thiel, S.Voss, Some experiences in solving the multiconstraint zero-one knapsack problems with Genetic Algorithm, INFOR 32, pp. 226-242,1994.

8. Mitsuo Gen, Runwei Cheng, (1996), A Survey of Penalty Techniques in Genetic Algorithms, IEEE International Conference on Evolutionary Techniques in Genetic Algorithms, pp.804-809

9. He Dakuo, Wang Fuli,(2002), Feedback penalty Function based on genetic Algorithm, In Proceedings of the $4^{\text {th }}$ World Congress on Intelligent Control and Automation, pp.,3153-3155.

10. Sangameswar Venkatraman, Gary G.Yen, (2004), A simple elitist Genetic Algorithm for Constrained Optimization, IEEE Congress on Evolutionary Computation (CEC2004), vol-1,Issue 19-23, pp.288295.

11. Simoes,A., Ernesto Costa (2000), Using Genetic Algorithms with Sexual or Asexual Transposition: a Comparative study, IEEE Proceedings of the 2000 Congress on Evolutionary Computing (CEC'2000). pp.1196-1203.

12. Simoes,A., Ernesto Costa (2003), Improving the Genetic Algorithm's Performance when using Transformation, In Proceedings of the Sixth International Conference on Artificial Neural Networks and Genetic Algorithms (ICANNGA'03), pp.175-181.

13. Alden H. Wright, Michael D. Vose, Kenneth A. De Jong, Lothar M. Schmitt, Foundations of Genetic Algorithms: 8th International Workshop, FOGA 2005, Aizu-Wakamatsu City, Japan, January 5-9, 2005 : Revised Selected Papers,Springer, 2005

14. Isibuchi.H \& Murata.T, Effects of repair process on the performance of EMO algorithms for multiobjective 0/1 knapsack problem, In Proceedings of the 2003 congress on Evolutionary Computation

15. Hitoshi Kanoh,. Yusuke Sakamoto, (2004 ) “ Interactive Timetabling System Using Knowledgebased genetic algorithms", IEEE International Conference on Systems man and Cybernetics (6), pp 5852-5857.

16. J.T. Richardson, M.R. Palmer, G. Lipins and M.Hilliard, "Some guidelines for Genetic algorithm with penalty functions, in Proceedings of the $3^{\text {rd }}$ International Conference on genetic algorithm, Morgan kauffman Publisher, CA, 1987, pp. 191-197.

17. Daniel Danciu, (2003),"Evolutionary Timetabling using Biased Genetic Operators", in Proceedings of the 25th International Conference on Information Technology Interfaces ITI 2003, june 16-19,Cavtat, Croatia.pp.477-482.
18. Sandy,B.Primrose, Richard,M., Twyman and Robert, W. Old, Principles of Gene Manipulation, $6^{\text {th }}$ edition

19. Bruce Alberts, Alexander Johnson, et.al, Molecular Biology of the Cell, $4^{\text {th }}$ edition.

20. Benjamin Lewin, GENES VIII, Molecular Biology Online edition, Virtual Tex

21. S.Martello, P. Toth, Knapsack problems: Algorithms and Computer Implementations, J.Wiley \& Sons, 1990. 